(C)2007 IEEE. Personal use of this material is permitted. However, permission to reprint/republish this material for advertising or promotional purposes or for creating new collective works for resale or redistribution to servers or lists, or to reuse any copyrighted component of this work in other works must be obtained from the IEEE 


\title{
Quantifying the Possible Financial Consequences of Failure for making a Risk Based Decision
}

\author{
Omar Khadeer Hussain, Elizabeth Chang, Farookh Khadeer Hussain and Tharam S. Dillon \\ Digital Ecosystem and Business Intelligence Institute, Curtin University of Technology, Perth, Australia \\ Omar.Hussain@postgrad.curtin.edu.au, \{Elizabeth.Chang,Farookh.Hussain,Tharam.Dillon\}@cbs.curtin.edu.au
}

\begin{abstract}
In a financial e-commerce interaction it is rational for the initiating agent to analyze beforehand the possible risk in interacting with any other agent. Doing so would give the initiating agent an idea of direction in which its interaction might head and also help it to make an informed decision of its future course of action with that particular agent. For risk analysis in the context of e-commerce interactions, the initiating agent has to determine beforehand the probability of failure and the possible consequences of failure in interacting with an agent. In this paper, we propose a methodology by which the initiating agent can determine beforehand the possible consequences of failure in its resources, according to the demand of its future interaction with an agent.
\end{abstract}

\section{Introduction}

Risk is an omnipresent factor in the modern world e-commerce interactions. It can be distinguished from other events due to the unwanted effects associated with it, and its ability to change the outcome of the interaction in a negative way or towards an unwanted direction. By specifying 'negative way' and 'unwanted direction' we mean the loss, or the occurrence of an undesired outcome in the interaction. In the literature researchers have defined risk by associating it with an unbiased outcome [1]. But the reality is that a positive event might not change the outcome of the interaction that is 'unwanted' in the interacting agent's view as much as the negative outcome does, and hence we think that risk is associated with the occurrence of negative outcomes. Risk analysis is important in the study of behavior in e-commerce interactions as there is a whole body of literature that argues that the decision to buy is based on the risk-adjusted costbenefit analysis [2]. Risk plays a central role in deciding whether to proceed with a transaction or not. It can broadly be defined as an attribute of decision making that reflects the variance of its possible outcomes. Thus, it commands a central role in any discussion that is related to a transaction. To assess the possible Risk in an interaction carried out in a particular context, it is first important to comprehend how risk is interpreted in that particular context. In the literature different definitions has been proposed for risk according to the context in which they are being discussed in [3-4]. Those definitions are defined according to how they fit and best expresses their object of analysis in that context. Similarly risk is assessed according to how it is defined in the particular context of analysis. The definition of risk and its assessment method of a context cannot be used to define and assess risk in other disciplines, as the way risk is interpreted and assessed in those disciplines varies and hence would give in-correct conclusions if applied. Therefore in the context of e-commerce interactions we interpret risk as a multidimensional construct which is a combination of the probability and level of failure of the interaction and the possible consequences of failure [5]. We term the initiating agent and the agent with whom it plans to interact with, as the risk assessing agent and the probable risk assessed agent respectively. It is possible that the risk assessing agent might have to choose an agent to interact with among a set of prospective agents, and to make an informed decision of its future course of interaction, it has to analyze the possible risk in interacting with each of them beforehand.

Risk analysis in interacting with an agent is done by ascertaining a) the probability of failure in achieving the desired outcomes; and $b$ ) the possible consequences of failure; in interacting with that agent. To make an informed risk based decision the risk assessing agent has to determine these two aspects to analyze and quantify the possible risk in interacting with each probable risk assessed agent. In our previous work we have developed a methodology by which the risk assessing agent can determine beforehand the probability of failure in interacting with a risk assessed 
agent according to the future demand of its interaction with it, by considering the various time in which its interaction is possible. In this paper, we propose a methodology by which the risk assessing agent can determine beforehand the possible consequences of failure in financial terms to its resources in interacting with a risk assessed agent. This paper is organized into 6 sections. In section 2 we give a brief overview of the methodology to determine the probability of failure in interacting with an agent. From section 3-5 we propose the methodology to determine the possible consequences of failure in an interaction and explain it with an example. Finally in section 6 we conclude the paper.

\section{Determining the Probability of Failure of an Interaction}

In this section we will give a brief overview of our previous work by which the risk assessing agent can determine beforehand the probability of failure in interacting with a risk assessed agent according to the future demand of its interaction with it. In order for the risk assessing agent to quantify the probability of failure in interacting with a risk assessed agent, we defined the term 'FailureLevel' and the Failure scale in Hussain et al. [5]. FailureLevel quantifies and semantically expresses the possible level of failure of the interaction, on the Failure scale. The Failure scale as shown in Figure 1 represents the different levels of failure, possible in a given period of time.

We propose that the risk assessing agent should determine beforehand the FailureLevel in interacting with the probable risk assessed agent, by ascertaining its in-capability to complete the interaction, according to its expectations or desired outcomes. In other words, the FailureLevel of an interaction is the extent to which the risk assessing agent determines that it might not achieve its desired outcomes in interacting with a probable risk assessed agent. The expectations or desired outcomes of the interaction show specifically what the risk assessing agent wants in its interaction with the risk assessed agent in the particular context. At a higher level, the expectations or the desired outcomes can be termed as the 'criteria' of the interaction. The risk assessing agent communicates the criteria of its interaction that it wants to achieve, and the resources it will invests to achieve them to the risk assessed agent before interacting with it, either in the expected behavior or the mutually agreed behavior. Hence by considering its expectations, the risk assessing agent accurately determines the probability

\begin{tabular}{|c|c|c|c|}
\hline $\begin{array}{l}\text { Semantics of } \\
\text { Failure Level }\end{array}$ & $\begin{array}{l}\text { Probability of } \\
\text { Failure }\end{array}$ & FailureLevels & Star Rating \\
\hline Unknown & - & -1 & Not Displayed \\
\hline Total Failure & $\begin{array}{l}91-100 \% \text { Probability of } \\
\text { Failure }\end{array}$ & 0 & Not Displayed \\
\hline Extremely High & $\begin{array}{c}71-90 \% \text { Probability of } \\
\text { Failure }\end{array}$ & 1 & 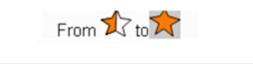 \\
\hline Largely High & $\begin{array}{c}51-70 \% \text { Probability of } \\
\text { Failure }\end{array}$ & 2 & 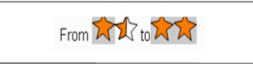 \\
\hline High & $\begin{array}{l}26-50 \% \text { Probability of } \\
\text { Failure }\end{array}$ & 3 & 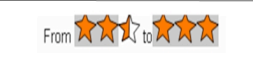 \\
\hline Significantly Low & $\begin{array}{l}\text { 11- } 25 \% \text { Probability of } \\
\text { Failure }\end{array}$ & 4 & From WWW tow \\
\hline Extremely Low & $\begin{array}{l}0-10 \% \text { Probability of } \\
\text { Failure }\end{array}$ & 5 & 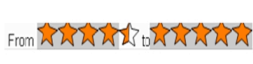 \\
\hline
\end{tabular}

Figure 1: The Failure scale

of failure according to its desired outcomes.

But as mentioned in the literature, risk is dynamic varying from time to time. As such, the risk assessing agent should take this dynamic nature of risk into consideration while undertaking risk analysis in interacting with a probable risk assessed agent. To incorporate that, we propose the risk assessing agent should divide the total time that it considers to determine the FailureLevel of the probable risk assessed agent, termed as the 'time space', into different non-overlapping parts, termed as 'time slots', and determine the FailureLevel of the risk assessed agent in each of those time slots. By doing so, the risk assessing agent ascertains the correct FailureLevel of the probable risk assessed agent in a time slot, according to its incapability to complete the criterions of its future interaction in that particular time slot, thus considering its dynamic nature while doing risk analysis. Further we propose that the risk assessing agent divides the time space of its interaction into two parts, called as the pre-interaction start time phase and post-interaction start time phase. 'Pre-Interaction start time phase' refers to the period of time before the risk assessing agent starts its interaction with the probable risk assessed agent, whereas 'Post-Interaction start time phase' is that period of time after the risk assessing agent commences and interacts with the probable risk assessed agent. The time slots in the time space will be spread out either in the pre-interaction or in the post-interaction start time phase. The risk assessing agent has to determine the FailureLevel of the probable risk assessed agent in each time slot according to the time phase in which they fall. The methodology for determining the FailureLevel of the probable risk assessed agent in both the pre- and postinteraction start time phase is defined in Hussain et al. $[5]$. 
After determining the FailureLevel of the risk assessed agent in each time slot of the post-interaction phase, we propose that the risk assessing agent compute its FailureLevel curve. This curve shows the different possible levels of failures while interacting with the risk assessed agent in the post-interaction time phase of the interaction. The abscissa of the FailureLevel curve gives the degree or level of failure and the corresponding ordinate or impulse gives the probability of occurrence of that level.

For better understanding, let us consider an example of risk assessing agent ' $A$ ' wanting to interact with a risk assessed agent ' $\mathrm{B}$ ' in context ' $\mathrm{C}$ ' for a period of 60 days. The criteria that it wants in the interaction are $\mathrm{C} 1$ and $\mathrm{C} 2$. Let us suppose that the risk assessing agent divides the time space into twenty equal time slots with ten in the pre-interaction time phase (t-10 till $\mathrm{t}-1)$ and ten in the post-interaction start time phase (t1 and t10) and uses the methodology defined in Hussain et al. [5] to determine the FailureLevel of the risk assessed agent in each time slot of the two phases of the time space. Let us suppose that from the FailureLevel obtained in each time slot of the post-interaction phase, the FailureLevel curve for the post-interaction period from time slot (t1 and t10) is as shown in figure 2 .

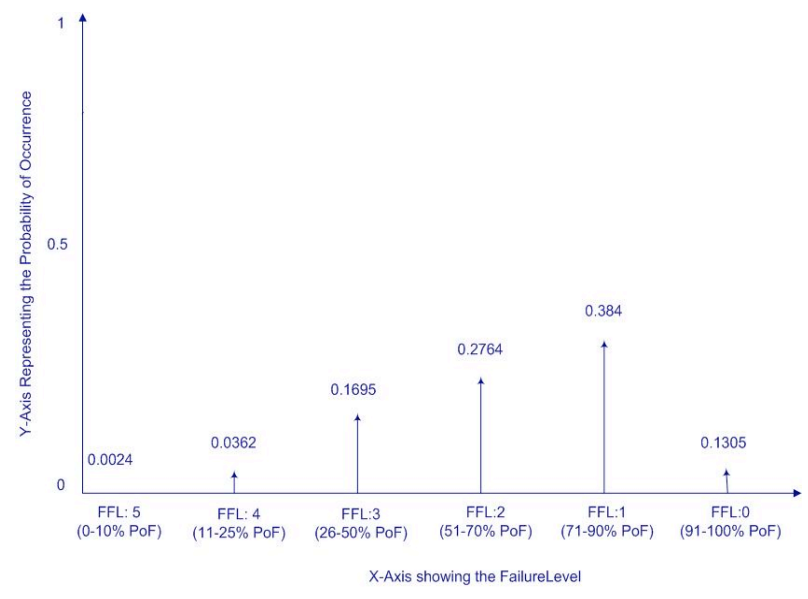

Figure 2: FailureLevel curve of the interaction

The above curve shows the probability of occurrence of each level of failure on the failure scale as determined by the risk assessing agent in interacting with a probable risk assessed agent, in the postinteraction time phase according to the criteria of its future interaction with it. Once the FailureLevel curve in interacting with a probable risk assessed agent has been determined, the risk assessing agent should then determine the net worth of resources that it going to invest in the interaction. In the next sections, we propose a methodology by which the risk assessing agent ascertains the net worth of its resources invested in an interaction, towards determining the possible consequences of failure to its resources.

\section{Determining the Amount Invested Curve}

The possible consequences of failure in an interaction are usually in the resources of the risk assessing agent that are involved in it. In a financial ecommerce interaction, we assume that the risk assessing agent interacts with the probable risk assessed agent to achieve its desired outcomes in exchange of the monetary financial value. Subsequently, in our context, the term 'resources' refers to the financial resources invested by the risk assessing agent in its interaction. In this paper we are interested in determining the possible consequences of failure to its financial resources involved in the interaction.

As mentioned earlier, the number of time slots in the post-interaction phase depends on the risk assessing agent's classification of the time space of the interaction. If there is more than one time slot in the post-interaction phase, then the net worth of the risk assessing agent's resources at stake in the interaction increases progressively, as the time slots increase according to the total worth invested in each of them. We are interested in determining the resources invested by the risk assessing agent only in the post-interaction phase of the interaction, as this is the phase in which the risk assessing agent will interact with the risk assessed agent and hence will put its resources at stake. To determine the net worth of its resources invested in the interaction, let us consider our previous discussion of the risk assessing agent ' $\mathrm{A}$ ' wanting to interact with a risk assessed agent ' $\mathrm{B}$ '. In its interaction there are ten time slots $(\mathrm{t} 1-\mathrm{t} 10)$ in the post-interaction time phase and let us suppose that the risk assessing agent ' $A$ ' invests $\$ 20,000$ in the interaction over those time slots. Two possibilities arise in the nature of the risk assessing agent investing its resources in the interaction. Either it is possible that the risk assessing agent invests the maximum threshold of its resources at once in the beginning of a time slot, or may invest it progressively, in stepwise way in each time slot. For example, it is possible that the risk assessing agent invests $\$ 20,000$; the total net worth of its resources in the first time slot, or it may invest the total worth of its resources progressively, that is say in the order of $\$ 2,000 ; \$ 3,000 ; \$ 6,000 ; \$ 5,000$ and $\$ 4,000$ in time slots $1,3,4,6$ and 9 of the post-interaction time phase respectively to gradually make the total worth of the 
interaction $\$ 20,000$. In both cases, the probability of an amount invested from its resources throughout the interaction is different. In the first case, when the risk assessing agent invests $\$ 20,000$ at the beginning of the first time slot, the net amount of its resources that it has at stake, throughout the time phase is $\$ 20,000$. On the other hand, if the resources were invested in a stepwise way in each time slot as explained earlier, then the total worth of its resources at stake reaches $\$ 20,000$ on time slot 19 of the time phase. This means that the risk assessing agent has $\$ 20,000$ worth of resources at stake for only 2 time slots of the time phase as compared to having $\$ 20,000$ at stake in all the time slots, in the first case. Hence, the risk assessing agent to determine the possible consequences of failure in its resources should first ascertain the accurate probability of the net worth of its resources at stake throughout the time space according to the investment nature of its resources.

To achieve that, we propose the calculation of an Amount Invested Curve (AIC). This curve gives the probability of an amount invested and at stake throughout the duration of the interaction from the invested resources of the risk assessing agent, to achieve its desired outcomes in interacting with a probable risk assessed agent, as initially decided in the expected or mutually agreed behavior. Another important property of this probabilistic model of the amount invested curve is that it describes the probability of the worth of the interaction to be at least a certain amount throughout the duration of the interaction. The essence of determining such a curve is to ascertain the consequences of failure in the interaction accurately according to net worth of the risk assessing agent's resources at stake.

To obtain the amount invested curve for our example, let us consider that the risk assessing agent invests $\$ 20,000$ in the interaction in a stepwise way as explained earlier. Determining and representing in figure 3 the amount invested curve for the interaction.

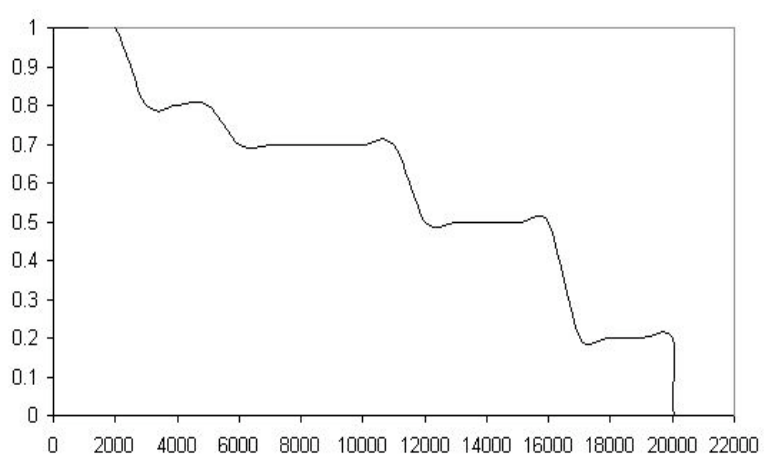

Figure 3: Amount invested curve for the interaction
It is possible that the risk assessing agent might not achieve its desired outcomes in interacting with a probable risk assessed agent in the resources initially promised, due to its FailureLevel resulting in the possible consequences of failure in its resources. The degree of the possible consequences of failure to the risk assessing agent's resources depends on the magnitude of the FailureLevel of the probable risk assessed agent. The higher the magnitude of failure of the probable risk assessed agent, the higher the possible consequences of failure to the risk assessing agent's resources and vice versa. The risk assessing agent, by determining the possible consequences of failure to its resources, apart from determining the FailureLevel in interacting with a probable risk assessed agent can ascertain the possible risk in interacting with it and subsequently can make an informed decision of its future course of action with that agent.

In the next sections, we will continue to propose the methodology by which the risk assessing agent can determine the possible consequences of failure to its resources in interacting with a probable risk assessed agent.

\section{Determining the Factual Amount Invested Curve}

To ascertain the possible consequences of failure in an interaction, we propose the calculation of the Factual Amount Invested Curve (FAIC). The factual amount invested curve shows the required probability of an amount to be kept at stake by the risk assessing agent throughout the duration of the interaction to achieve its desired outcomes, by taking into consideration the FailureLevel of the risk assessed agent, and the probability of that amount it was initially investing. Hence, the factual amount invested curve (FAIC), which shows the increased probability of an amount that the risk assessing agent needs to invests in an interaction, is an extension of the amount invested curve (AIC). The AIC shows the actual probability of an amount invested and at stake from the resources of the risk assessing agent throughout the duration of the interaction, according to the expected or mutually agreed behavior, whereas the FAIC shows the required probability of the risk assessing agent to invest that amount throughout the duration of the interaction by considering the FailureLevel of the risk assessed agent.

To obtain the FAIC of an interaction, the AIC should be convolved with the FailureLevel curve of the 
risk assessed agent. We utilize the cumulants method for the convolution of these two functions. In this method, the convolution of the independent random variables can be expressed as a sum of their individual cumulants, which can then be used to model the output curve, which is the FAIC by using either GramCharlier series expansion or Beta distribution. In our problem, an advantage of using this method over the conventional point by point method for convolution is that, in the cumulants method the convolution of the independent random variables can be determined as a sum of their individual cumulants, whereas in the conventional method it is necessary to divide the AIC into a number of parts and then convolve them with the density function of the FailureLevel curve of the risk assessed agent on each point it. The output of the convolution, which is the FAIC, is an inflated curve as compared to the AIC. This curve is inflated as it shows the increased probability of an amount that the risk assessing agent needs to invest in the interaction.

Considering the previous example, the risk assessing agent ' $A$ ' can determine the FAIC in time slots $\mathrm{t} 1$ - t10 of its interaction, by convolving the FailureLevel curve of the risk assessed agent in those time slots, represented in figure 2 with the AIC of those time slots represented in figure 3 . The resultant inflated FAIC of the interaction is given in figure 4 .

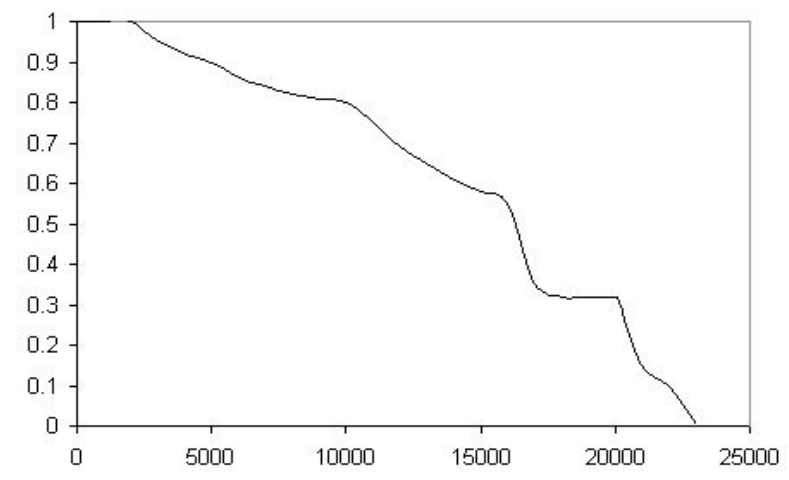

Figure 4: Factual Amount invested curve for the interaction

Once the risk assessing agent determines the FAIC, it can then determine the possible consequences of failure to its resources in interacting with the particular risk assessed agent.

\section{Determining the Possible Consequences of Failure in an Interaction}

To risk assessing can determine the possible consequences of failure to its resources in an interaction, by ascertaining the level of uncommitted behaviour in financial terms by the probable risk assessed agent in the interaction. The level of uncommitted behaviour in the interaction is the level to which the probable risk assessed agent will not complete the desired outcomes of the risk assessing agent in the time and resources as promised earlier. The risk assessing agent can ascertain this level of uncommitted behaviour in financial terms of its resources by using the Amount and the Factual Amount Invested Curve, i.e. AIC and FAIC respectively. By the definition of these curves, AIC shows the probability of an amount invested by the risk assessing agent as decided initially, to achieve its desired outcomes. At the end of the AIC the risk assessing agent expects to achieve its desired outcomes of the interaction according to the expected or mutually agreed behavior, in the resources it invested. FAIC shows the required probability of an amount to be invested by the risk assessing agent to achieve its same desired outcomes, by considering the FailureLevel of the risk assessed agent in the interaction. As opposed to what was promised initially, it is possible that at the end of AIC, the risk assessing agent still has some desired outcomes to achieve, due to the FailureLevel of the risk assessed agent. We term the level to which the probable risk assessing agent will not achieve its desired outcomes in financial terms as the loss of its investment probability (LOIP). Therefore the LOIP index in the interaction is simply the ordinate on the FAIC, immediately after the end of the net resources invested in the interaction, i.e. at the end of AIC. Because by definition of FAIC, this ordinate is the probability of its corresponding amount needed to be at stake in the interaction, but this amount will not invested by the risk assessed agent as it is more than what was initially agreed upon. Hence

$$
\mathrm{LOIP}=\mathrm{FAIC}(\mathrm{w})
$$

where, $w$ is the abscissa immediately after the end of $\mathrm{AIC}$, and

FAIC $(w)=$ Ordinate of the Factual amount invested curve at point ' $\mathrm{w}$ ' which is after investing the total resources of the interaction.

The possible consequences of failure of an interaction can then be determined by the accumulated un-committed behavior in financial terms in the interaction. This accumulated un-committed behaviour of the interaction is that behaviour which theoretically will not be met by the probable risk assessed agent in the time and resources promised initially, resulting in the loss in the resources of the risk assessing agent. Hence the possible consequences of failure in an 
interaction is proportional to the area under the FAIC beyond $\mathrm{w}$, which is the point the risk assessing agent invests the total worth of its resources in the interaction, i.e. the end of the AIC. Subsequently, the possible consequence of failure (PCF)

$$
\mathrm{PCF}=\int_{w}^{\alpha} \operatorname{FAIC}(\mathrm{x}) \mathrm{dx}
$$

The possible consequences of failure in an interaction will be proportional to the area of the curve under the FAIC, after the point $\mathrm{w}$. The greater the area of the FAIC curve after point w, higher will be the possible consequences of failure and vice versa.

Extending the previous example, the risk assessing agent ' $A$ ' can utilize the methodology to determine the loss of its investment probability (LOIP) in interacting with the risk assessed agent ' $\mathrm{B}$ ' in the time slots of its interaction. In the post-interaction time phase of its interaction the risk assessing agent ' $A$ ' invests its resources in a stepwise way to achieve its desired outcomes. But as shown in figure 5 at the end of the net resources invested, that is, at the end of AIC, the LOIP index of the interaction is 0.32 , which means that there is $32 \%$ probability of loss in the risk assessing agent's resources invested in the interaction due to its FailureLevel and as a result un-fulfillment of its desired outcomes, as opposed to what was promised initially. Subsequently the possible consequence of failure in the interaction is the area under the FAIC, from the end of AIC.

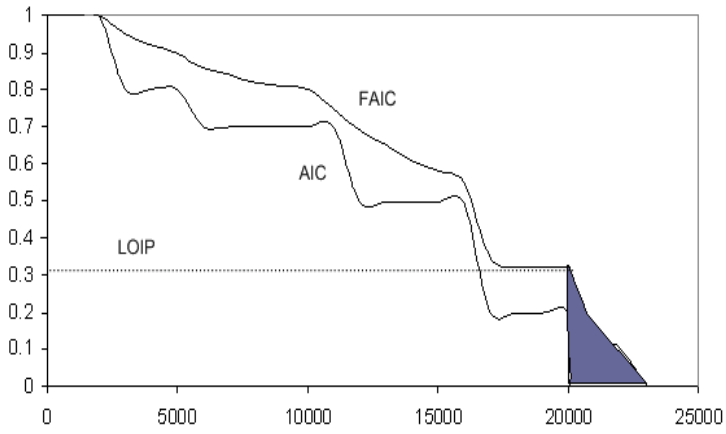

Figure 5: Possible consequences of Failure in an Interaction

\section{Conclusion}

In this paper we proposed a methodology by which the risk assessing agent can determine beforehand the possible consequences of failure to its resources and the loss in its investment probability in interacting with a probable risk assessed agent. The risk assessing agent by considering the probability of failure and the possible consequences of failure in interacting with a risk assessed agent can analyze the possible risk in interacting with it and subsequently based on that, it can make an informed decision of its future course of action what that agent.

\section{References}

[1] R.C. Mayer, J.H. Davis and F.D. Schoorman, "An interactive model of organizational trust", Academy of Management Review, vol. 20, no. 3, 1995, pp.709-734.

[2] S. Greenland, "Bounding analysis as an inadequately specified methodology", Risk Analysis vol. 24, no. 5, 2004, pp. 1085-1092.

[3] N. Luhmann, "Familiarity, confidence, trust: Problems and alternatives", Trust: Making and Breaking Cooperative Relations, Basil Blackwell, New York, USA, 1988.

[4] D.M. Rousseau, S.B. Sitkin, R.S. Burt and C. Camerer, "Not so different after all: A cross-discipline view of trust", Academy of Management Review, vol. 23, no. 4, 1998, pp. $393-404$

[5] O. K. Hussain, E. Chang, F. K. Hussain and T. S. Dillon, "A methodology to quantify failure for risk-based decision support system in Digital Business Ecosystems", Data \& Knowledge Engineering, Elsevier Science (Article in Press). 